\title{
Methods of Synthesis of Quasi - Balanced Circuits for Measuring of Impedance Components
}

\author{
Adam Cichy ${ }^{1}$ \\ ${ }^{I}$ Institute of Measurement Science, Electronics and Control, Silesian University of Technology, \\ Krzywoustego St. 10, 43-150 Gliwice, Poland \\ adam.cichy@polsl.pl
}

\begin{abstract}
Quasi-balanced circuits are not widely known and often built. This is mainly due to insufficient knowledge of their properties. Meanwhile, these systems have properties which may be their great advantages. Quasi-balanced circuits have a maximum convergence, allowing the full independent quasi-balancing for each of the components. Although the simple quasi-balanced circuits enable the measurement of a single component, but there are solutions which allow the measurement of the impedance of the two components at the same time. The article presents two methods of the synthesis of quasi-balanced circuits, which are different in approach. The first method is based on a linear model of the transducer, the other on the analysis of the measuring signals of quasibalanced bridges. Virtual instrument was implemented and the results of measurements of selected elements were compared with results from other device. This confirms the usefulness of the method.
\end{abstract}

Index Terms-Bridge circuits; capacitance measurement; circuit synthesis; impedance measurement.

\section{INTRODUCTION}

Quasi-balanced measuring circuits are used to measure the impedance components. Like the balanced circuits they require the circuit to bring the selected state. Unlike the balanced circuits, this occurs by changing settings of a single regulatory element, which allows measuring one component of the impedance [1]-[4]. There are quasibalanced circuits devoid of these disadvantages - circuits with dual quasi-balancing and parallel quasi-balanced circuits [5]-[7]. The selected non-zero state of the circuit is called a state of quasi - equilibrium. Most often, it is selected phase angle between two selected signals of the system. These circuits are not often described in the literature. They are characterized by an advantageous feature - a typical convergence problem of the known balanced circuits does not exist in quasi-balanced circuits.

\section{PRINCIPLE OF OPERATIONS}

In quasi-balanced circuits the phase-angle between two selected signals $w_{1}$ and $w_{2}$ is a subject to the detection. This signal is connected to the phase detector, which detects the quasi-equilibrium state. Structural diagram of signal processing in the quasi-balanced circuits is shown in Fig. 1, wherein $\mathrm{Z}$ is a block, which produces measurement signals $V_{X}$ and $I_{X}$, containing the measured impedance $\mathrm{Z}$ and a power source, $V_{X}, I_{X}$ are signals representing the current and voltage signals of the impedance $Z, \mathrm{~T}$ is signal processing block (transducer) and PD is a phase detector.

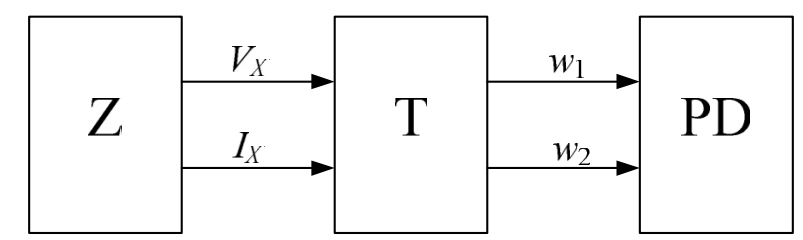

Fig. 1. General structural diagram of a quasi-balanced circuit.

A similar structure with regard to the balanced circuits has been presented in [4].

The concept of impedance $\mathrm{Z}$ is the ratio of two-terminal circuit describing the composite signals of voltage $V_{X}$ and current $I_{X}$

$$
Z=\frac{V_{X}}{I_{X}}
$$

Block $\mathrm{T}$ is a linear transducer, whose diagram is shown in Fig. 2. Its linearity results from the selection of quasiequilibrium signal as the phase angle between two sinusoidal signals $w_{1}$ and $w_{2}$.

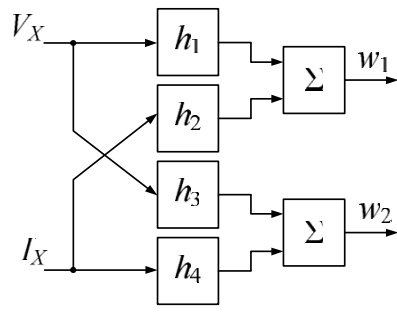

Fig. 2. Structure of the transducer T.

The $w_{1}$ and $w_{2}$ signals are formed in the block $\mathrm{T}$ as a function of complex signals $V_{X}$ and $I_{X}$, according to the processing equation:

$$
\left\{\begin{array}{l}
w_{1}=h_{1} V_{X}+h_{2} I_{X}, \\
w_{2}=h_{3} V_{X}+h_{4} I_{X},
\end{array}\right.
$$

where $h_{1}, h_{2}, h_{3}, h_{4}$ are complex parameters of the 
transmitter T. Below a method that allows the synthesis of quasi-balanced circuits using a model of Fig. 2 has been described.

\section{SyNTHESIS BASED ON A MATHEMATICAL MODEL}

Rearranging (2) we get

$$
\frac{w_{1}}{w_{2}}=\frac{h_{1} V_{X}+h_{2} I X}{h_{3} V_{X}+h_{4} I_{X}}=\frac{h_{1} Z_{X}+h_{2}}{h_{3} Z_{X}+h_{4}},
$$

Equation (3) can be further represented as follows

$$
\frac{w_{1}}{w_{2}}=\frac{\left(h_{1} Z_{X}+h_{2}\right)\left(\overline{h_{3} Z_{X}+h_{4}}\right)}{\left|h_{3} Z_{X}+h_{4}\right|^{2}} .
$$

The detected signal $\Phi$ in quasi - balanced circuits is mostly a phase angle between the selected signals $w_{1}$ and $w_{2}$. The signal $\Phi$ is the argument of the $w_{1} / w_{2}$ ratio and is described by the equation

$$
\Phi=\operatorname{Arg}\left(\frac{w_{1}}{w_{2}}\right)
$$

Equation (4) after taking into account (5) can be written as follows

$$
\Phi=\operatorname{Arctan} \frac{\operatorname{Im}\left[\left(h_{1} Z_{X}+h_{2}\right)\left(\overline{h_{3} Z_{X}+h_{4}}\right)\right]}{\operatorname{Re}\left[\left(h_{1} Z_{X}+h_{2}\right)\left(\overline{h_{3} Z_{X}+h_{4}}\right)\right]} .
$$

In the quasi-equilibrium state the signal $\Phi$ has a predetermined value $\Phi_{q}$

$$
\Phi=\Phi_{q}
$$

In this case, assuming

$$
\tan \Phi_{q}=q
$$

equation (7) can be written in the following form

$$
q=\frac{\operatorname{Im}\left[\left(h_{1} Z_{X}+h_{2}\right)\left(\overline{h_{3} Z_{X}+h_{4}}\right)\right]}{\operatorname{Re}\left[\left(h_{1} Z_{X}+h_{2}\right)\left(\overline{h_{3} Z_{X}+h_{4}}\right)\right]} .
$$

Blocks with transmittances $h_{1}, h_{2}, h_{3}, h_{4}$ may be constructed as shown in Fig. 3.

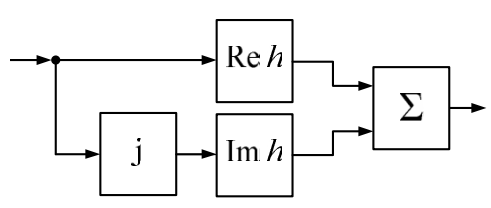

Fig. 3. Structure of transmitations $h_{1}, h_{2}, h_{3}, h_{4}$.

After transformation of (9) is obtained by the equation

$$
a_{1}\left|Z_{X}\right|^{2}+a_{2} \operatorname{Re} Z_{X}+a_{3} \operatorname{Im} Z_{X}+a_{4}=0,
$$

where:

$$
\left\{\begin{array}{l}
a_{1}=q \operatorname{Re}\left(h_{1} \overline{h_{3}}\right)-\operatorname{Im}\left(h_{1} \overline{h_{3}}\right), \\
a_{2}=q \operatorname{Re}\left(h_{1} \overline{h_{4}}+h_{2} \overline{h_{3}}\right)-\operatorname{Im}\left(h_{1} \overline{h_{4}}+h_{2} \overline{h_{3}}\right), \\
a_{3}=\operatorname{Re}\left(h_{1} \cdot \overline{h_{4}}-h_{2} \overline{h_{3}}\right)-q \operatorname{Im}\left(h_{1} \overline{h_{4}}-h_{2} \overline{h_{3}}\right), \\
a_{4}=q \operatorname{Re}\left(h_{2} \overline{h_{4}}\right)-\operatorname{Im}\left(h_{2} \overline{h_{4}}\right) .
\end{array}\right.
$$

Equation (10) shows the general processing equation of the circuit in which the transducer $\mathrm{T}$ has the form shown in the diagram in Fig. 3. The components of impedance can be determined from equation (11) subject to the following conditions:

1. the coefficients $a_{1}, a_{2}, a_{3}, a_{4}$ appearing in (10), by components other than the measured component, must be equal to zero,

2. the equation, allowing determine the measured impedance component, should have the simplest form.

Let us consider the possibility of building the system to measure the reactive component of impedance RC. Suppose the serial model. The fulfillment of condition 1 means that

$$
\left\{\begin{array}{l}
a_{1}=q \operatorname{Re}\left(h_{1} \overline{h_{3}}\right)-\operatorname{Im}\left(h_{1} \overline{h_{3}}\right)=0 \\
a_{2}=q \operatorname{Re}\left(h_{1} \overline{h_{4}}+h_{2} \overline{h_{3}}\right)-\operatorname{Im}\left(h_{1} \overline{h_{4}}+h_{2} \overline{h_{3}}\right)=0 .
\end{array}\right.
$$

The equation allowing determine the measured impedance component will then have the form

$$
\begin{gathered}
\operatorname{Im} Z_{X}=-\frac{a_{4}}{a_{3}}= \\
=-\frac{q \operatorname{Re}\left(h_{2} \overline{h_{4}}\right)-\operatorname{Im}\left(h_{2} \overline{h_{4}}\right)}{\operatorname{Re}\left(h_{1} \cdot \overline{h_{4}}-h_{2} \overline{h_{3}}\right)-q \operatorname{Im}\left(h_{1} \overline{h_{4}}-h_{2} \overline{h_{3}}\right)} .
\end{gathered}
$$

The most commonly used quasi-balance state is the state of orthogonality of signals $w_{1}$ and $w_{2}$. Moreover, the analysis of (12) and (13) follows that the equations are simplified considerably when the parameter $h_{3}$ equals 0 . Equation (12) is the fulfilled, e.g. when the parameter $h_{1}$ is a real number, and $h_{2}$ and $h_{4}$ parameters are purely imaginary numbers and they are equal each other. The structure of such a circuit is shown in Fig. 4.

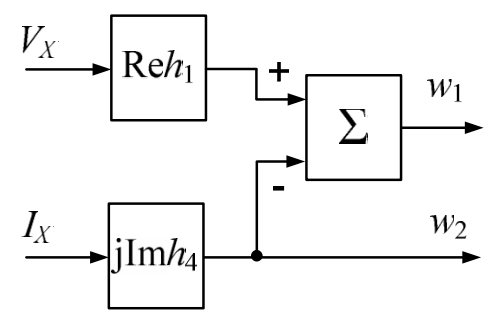

Fig. 4. Diagram of quasi-balanced bridge with phase detector for passive component of capacitor impedance.

The equation to determine the capacity will have in the system form

$$
C_{X}=\frac{\operatorname{Im} h_{2}}{\omega \operatorname{Re} h_{1}} .
$$




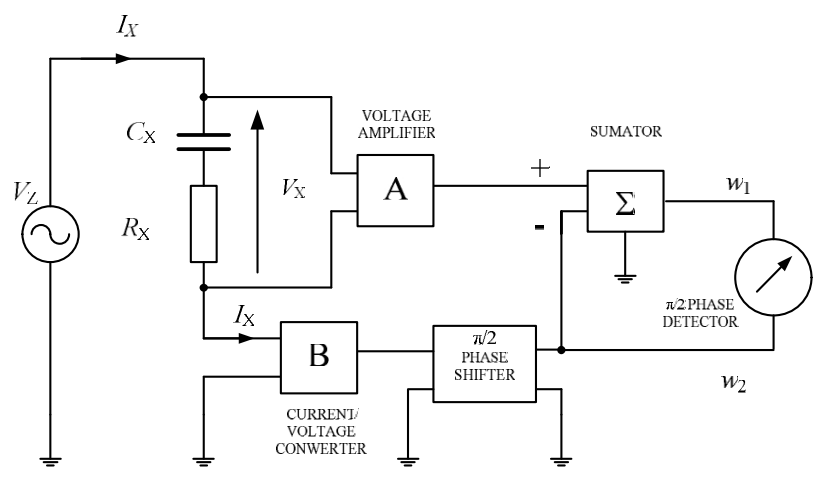

Fig. 5. Diagram of quasi-balanced circuit for capacitance measurement.

An example of realisation of the circuit from Fig. 4 has been shown on Fig. 5 [8].

\section{SYNTHESIS BASED ON A BRIDGE CIRCUIT}

Synthesis of quasi-balanced circuits can also be conducted based on an analysis of the known bridge circuits. Fig. 6 shows the bridge quasi-balanced circuit designed to measure the capacity of the serial object of RC type [4].

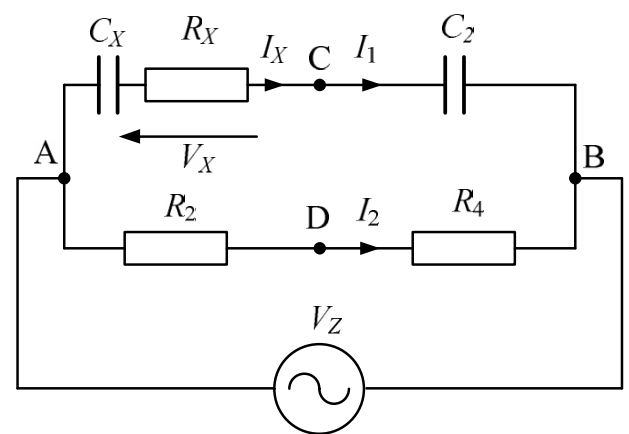

Fig. 6. Diagram of quasi-balanced bridge with phase detector for real component of capacitor impedance.

In this circuit, assumed as a state of quasi-equilibrium the orthogonality of the selected voltages $V_{D C}$ and $V_{C B}$

$$
V_{D C} \perp V_{C B}
$$

This state means that

$$
\operatorname{Re} \frac{V_{D C}}{V_{C B}}=0
$$

Figure 7 shows the vector diagram of voltages and currents of the bridge of Fig. 6.

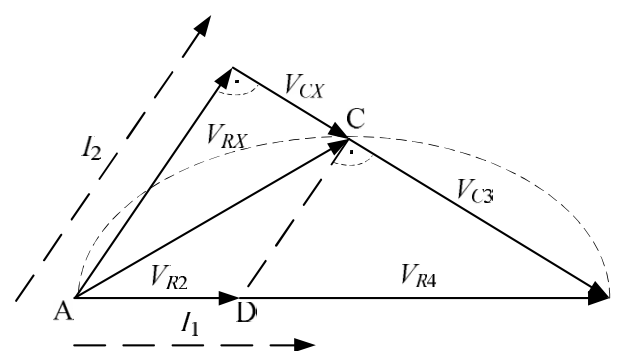

Fig. 7. Vector diagram of the bridge from Fig. 6.

The classic approach to the analysis of the circuit of Fig. 6 is a geometric approach. According to the theorem of Thales, ratios of appropriate RMS values of voltages read from the phasor diagram shown in Fig. 7, must meet the proportion of

$$
\frac{V_{C X}}{V_{C 3}}=\frac{V_{R 2}}{V_{R 4}} .
$$

The RMS voltages in (17) are the products of RMS values of currents and resistances or reactances of appropriate circuit elements, and therefore the equation can be written as follows

$$
\frac{I_{1} \operatorname{Im} Z_{X}}{I_{1} \operatorname{Im} Z_{3}}=\frac{I_{2} R_{4}}{I_{2} R_{2}} .
$$

After reduction and transformation of (18) can be written as follows

$$
\operatorname{Im} Z_{X}=\operatorname{Im} Z_{3} \frac{R_{4}}{R_{2}},
$$

where, taking into account that

$$
\operatorname{Im} Z_{X}=\frac{1}{\omega C_{X}},
$$

the measured capacitance $C_{X}$ can be determine according to the following equation

$$
C_{X}=C_{3} \frac{R_{2}}{R_{4}} .
$$

Bridge's selected signals, as already mentioned, are the voltages $V_{D C}$ and $V_{C B}$ :

$$
\left\{\begin{array}{l}
V_{D C}=-I_{1} \frac{1}{j \omega C_{3}}+I_{2} R_{4}, \\
V_{C B}=\frac{1}{j \omega C_{3} R_{3}} I_{1} .
\end{array}\right.
$$

Taking into account the indications of Fig. 2, after transformations, (22) can be written:

$$
\left\{\begin{array}{l}
w_{1}=\left(\frac{R_{4}}{R_{2}+R_{4}}\right) V_{X}-\left(\frac{R_{2}}{j \omega C_{3}\left(R_{2}+R_{4}\right)}\right) I_{X}, \\
w_{2}=\frac{1}{j \omega C_{3}} I_{X},
\end{array}\right.
$$

where we can determine coefficients of the model in Fig. 2:

$$
\left\{\begin{array}{l}
h_{1}=\frac{R_{4}}{R_{2}+R_{4}}, \\
h_{2}=-\frac{R_{2}}{j \omega C_{3}\left(R_{2}+R_{4}\right)}, \\
h_{3}=0 \\
h_{4}=\frac{1}{j \omega C_{3}} .
\end{array}\right.
$$

It may be noted that (24) shows the layout of the block 
diagram shown in Fig. 4, with the same processing equation.

\section{TESTING}

Figure 5 shows the structure of the system derived using the methods described above. The discussed system has been implemented as a virtual instrument. The virtual structure allows for a simulated testing of the measuring system using artificially generated measurement signals. Then it is possible to test exactly the same structure using the real measurement signals. The virtual instrument was implemented using the LabView [9] package. The structure of the virtual instrument is shown in Fig. 7.

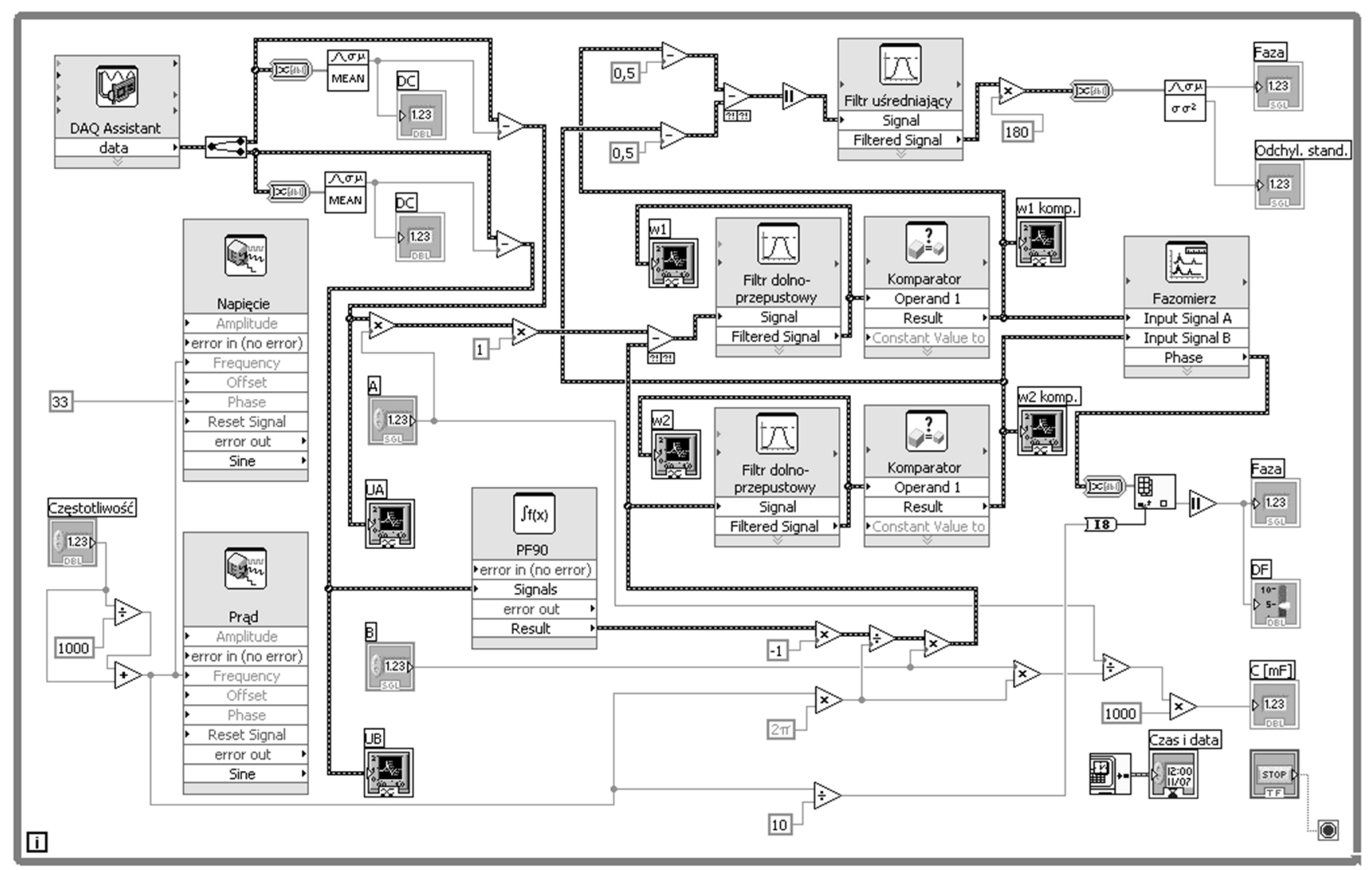

Fig. 7. The structure of virtual instrument.

Typical capacitors range from $1 \mathrm{nF}$ to $1 \mu \mathrm{F}$ were used as tested objects. The measurement signals are the voltage across the capacitor and the voltage drop across the shunt that is proportional to the current of the capacitor under test. The signals are given to the input of the NI 6251 data acquisition card [10]. Figure 8 shows a view of the measuring system.

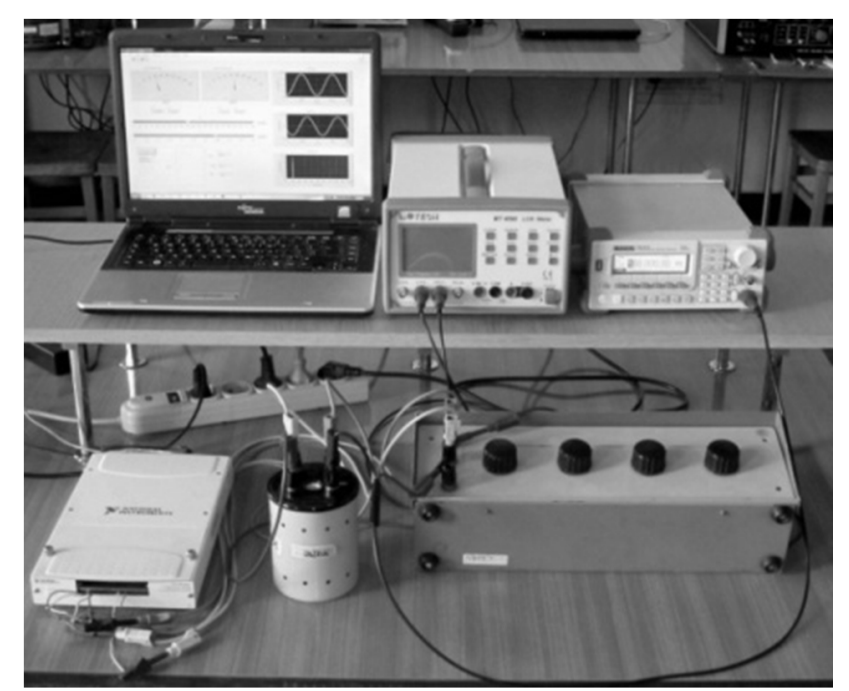

Fig. 8. View of the test stand.
The structure of Fig. 7 is versatile and other quasibalanced circuits can be very easy implemented on it. On the test stand of Fig. 8 it is possible to test various virtual instruments [11]-[12].

Test results were compared with the indications of the Agilent E4980A Precision LCR Meter [13]. The results are shown in Table I and in Fig. 9. Errors associated with the phase detection and relatively low input resistances of the DAQ are primarily the sources of errors of the realized instrument.

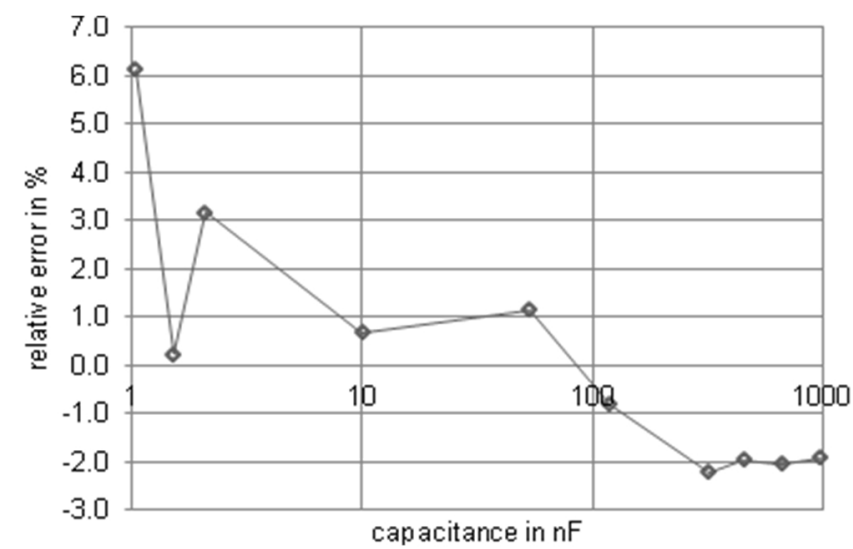

Fig. 9. The graph of the error of the realized circuit. 
TABLE I. RESULTS OF COMPARING

\begin{tabular}{|c|c|}
\hline Agilent E4980A, $\mathbf{n F}$ & Realized circuit, $\mathbf{n F}$ \\
\hline 1.030 & 1.10 \\
\hline 1.503 & 1.51 \\
\hline 2.053 & 2.12 \\
\hline 9.949 & 10.02 \\
\hline 53.581 & 54.20 \\
\hline 119.461 & 118.50 \\
\hline 319.279 & 312.34 \\
\hline 455.326 & 446.54 \\
\hline 665.323 & 652.04 \\
\hline 979.042 & 960.65 \\
\hline
\end{tabular}

Sources of error of the realized instrument are primarily errors associated with the phase detection and a relatively low input resistance of the DAQ.

\section{CONCLUSIONS}

The article presents two methods for the synthesis of quasi-balanced circuits for measuring the impedance components.

The first method uses a processing model of current and voltage signals of the impedance under test. It is a general method, employing the general processing equation of quasi- balanced circuits. Using this method, the system for capacitance measuring has been derived.

The second method is based on an analysis of the known bridge circuits. The processing equation of the quasibalanced bridge circuit has been derived, and then built the circuit's layout. In both cases, the same system has been derived.

The test results confirm the usefulness of the measurement method, derived in the previous section. The prototype measuring system is characterized by the errors of a few percent compared to the precision instrument Agilent, that in further work will be certainly improved.

Quasi-balanced circuits can become complementary to known measurement systems. Their undeniable advantage is maximum convergence. They are easy to implement, especially as virtual instruments.

\section{REFERENCES}

[1] H. Amira, M. Hfaiedh, M. Valentin, "Quasi-balanced bridge method for the measurements of the impedances", IET Sci. Meas. Technol, vol. 3, no. 6, pp. 403-409, 2009. [Online]. Available: http://dx.doi.org/10.1049/iet-smt.2009.0032

[2] M. I. Burbelo, "Universal quasi-balanced bridges for measuring the parameters of four-element two-terminal networks", Measurement Techniques, vol. 44, no. 11, pp. 1130-1133, 2001. [Online]. Available: http://dx.doi.org/10.1023/A:1014017418854

[3] M. A. Atmanand, V. Jagadeesh Kumar, V. G. K. Murti, "A microcontroller based quasi-balanced bridge for the measurement of $\mathrm{L}, \mathrm{C}$ and R", IEEE Trans. on Instrum. \& Meas., vol. 45, no. 3, pp. 15, 1996. [Online]. Available: http://dx.doi.org/10.1109/19.494595

[4] K. B. Karandeev, Bridge and potentiometer methods of electrical measurements. Moscow: Peace Publishers, 1966.

[5] A. Cichy, "Non-bridge circuit with double quasi-balancing for measurement of dielectric loss factor", IET Sci. Meas. Technol., vol. 7, no. 5, pp. 274-279, 2013. [Online]. Available: http://dx.doi.org/10.1049/iet-smt.2012.0130

[6] A. Cichy, A. Skorkowski, S. Barwinek, "Double quasi-balanced meter for measurement of inductor quality factor", 19th Symposium IMEKO TC-4 Symposium and 17th TC-4 IWADC. Workshop Advances in Instrumentation and Sensors Interoperability, Barcelona, 2013.

[7] A. Cichy, "Possibility of building parallel quasi-balanced circuits and their realizations", Przeglad Elektrotechniczny, vol. 89, no. 4, pp. 217-220, 2013.

[8] A. Skorkowski, A. Cichy, "Virtual capacitance meter based on impedance modulus measurement", in Proc. XIX IMEKO World Congress, Lisbon, Portugal, 2009.

[9] LabVIEW system design software. [Online] Available: http://www.ni.com/labview

[10] LabVIEW system design software. [Online] Available: http://www.ni.com/pdf/manuals/371291h.pdf

[11] A. Cichy, A. Skorkowski, "Virtual system for quality factor measurements of coils uses quasi-balanced method of impedance components measurements", Przeglad Elektrotechniczny, vol. 89, no. 01a, pp. 88-91, 2013.

[12] A. Cichy, A. Skorkowski, S. Barwinek, "Automated quasi-balancing in virtual quasi-balanced circuit designed to capacitance measurements", 19th Symposium IMEKO TC-4 Symposium and 17th TC-4 IWADC. Workshop Advances in Instrumentation and Sensors Interoperability, Barcelona, 2013.

[13] Keysight Technologies E4980A Precision LCR Meter $20 \mathrm{~Hz}$ to 2 MHz E4980AL Precision LCR Meter $20 \mathrm{~Hz}$ to $300 \mathrm{kHz} / 500 \mathrm{kHz} / 1$ $\mathrm{MHz}$ Data Sheet. [Online] Available: http://iterature.cdn.keysight.com/litweb/pdf/5989-4435EN.pdf 